\title{
Two Kinds of Darboux-Bäcklund Transformations for the $q$-Deformed KdV Hierarchy with Self-Consistent Sources
}

\author{
Hongxia Wu, ${ }^{1,2}$ Liangjuan Gao, ${ }^{1} J^{\prime}$ gxin Liu,, ${ }^{1}$ and Yunbo Zeng ${ }^{3}$ \\ ${ }^{1}$ Department of Mathematics, School of Sciences, Jimei University, Xiamen 361021, China \\ ${ }^{2}$ Department of Mathematics, The University of Texas Rio Grande Valley, Edinburg, TX 78539, USA \\ ${ }^{3}$ Department of Mathematics, Tsinghua University, Beijing 100084, China \\ Correspondence should be addressed to Hongxia Wu; wuhongxia@jmu.edu.cn
}

Received 19 April 2016; Accepted 23 July 2016

Academic Editor: Pavel Kurasov

Copyright (C) 2016 Hongxia Wu et al. This is an open access article distributed under the Creative Commons Attribution License, which permits unrestricted use, distribution, and reproduction in any medium, provided the original work is properly cited.

\begin{abstract}
Two kinds of Darboux-Bäcklund transformations (DBTs) are constructed for the $q$-deformed $N$ th KdV hierarchy with selfconsistent sources ( $q$-NKdVHSCS) by using the $q$-deformed pseudodifferential operators. Note that one of the DBTs provides a nonauto Bäcklund transformation for two $q$-deformed $N$ th KdV equations with self-consistent sources $(q$-NKdVESCS) with different degree. In addition, the soliton solution to the first nontrivial equation of $q$-KdVHSCS is also obtained.
\end{abstract}

\section{Introduction}

The $q$-deformed integrable systems are regarded as the $q$ deformation of the related classical ones. The $q$-deformation is performed by using the $q$-derivative $\partial_{q}$ to take the place of usual derivative $\partial_{x}$ and it reduces to a classical integrable system as $q \rightarrow 1$. In recent years, some $q$-deformed integrable systems, especially the $q$-deformed $N$ th KdV hierarchy ( $q$ $\mathrm{NKdVH})$ and the $q$-deformed KP hierarchy $(q-\mathrm{KPH})$, have attracted much interest both in mathematics and in physics [1-17]. It was shown that $q$-NKdVH inherited some integrable structures from the classical $\mathrm{Nth} \mathrm{KdV}$ hierarchy, such as infinite conservation law [2], bi-Hamiltonian structure [3,4], tau function [5, 6], Darboux-Bäcklund transformation [7], and $q$-Miura transformation [8]. In 1999, some elementary DBTs of the $q$-NKdVH (also called $q$-deformed GelfandDickey hierarchy) were constructed by using the $q$-deformed pseudodifferential operators. The formula for the $n$-times repeated DBTs was also presented, which produces the new soliton solutions to the $q-\mathrm{NKdVH}$ [7]. For $q-\mathrm{KPH}$, its biHamiltonian structure, tau function, additional symmetries, $q$-effect in $q$-soliton, Virasoro constraints of tau function and integrable extension, and $q$-Bäcklund transformation were also explored in [9-17]. In 2008, based on the symmetry constraint for $q-\mathrm{KPH}$, the new extension of this hierarchy was considered [16]. Two kinds of reductions of this new extended $q$-KP hierarchy were also studied, which give many $1+1$ dimensional $q$-deformed soliton equations with selfconsistent sources [16]. For example, the $n$-reduction $L_{<0}^{n}=0$ gives $q$-NKdVHSCS. However, to our knowledge, the DBTs and the soliton solution for $q$-NKdVHSCS still remain unexplored. It is known that the DBT is an important property to characterize the integrability of the hierarchy. Thus, it is necessary for us to explore the DBT for $q$-NKdVHSCS. We think our research results will deepen our understanding on soliton solutions of this hierarchy.

The outline of this paper is as follows. In Section 2, some notations in the $q$-calculus and the definition of the $q$ NKdVHSCS are briefly reviewed. In Section 3, we aim at the construction of auto DBTs for $q$-NKdVHSCS. In Section 4, the nonauto DBTs for $q$-NKdVHSCS are constructed. In Section 5 , one soliton solution to the first nontrivial equation of $q$-NKdVHSCS is obtained by using nonauto DBTs. Section 6 is devoted to a brief summary.

\section{The $q$-Deformed $N$ th KdV Hierarchy with Self-Consistent Sources ( $q$-NKdVHSCS)}

In this section, we briefly review some notations in the $q$ calculus and the definition of the $q$-NKdVHSCS. 
The $q$-derivative operator $\partial_{q}$ and $q$-shift operator $\theta$ are defined by

$$
\begin{aligned}
{\left[\partial_{q} f(x)\right] } & =\frac{f(q x)-f(x)}{x(q-1)}, \\
\theta(f(x)) & =f(q x) .
\end{aligned}
$$

In this paper, we introduce two notations: $[P f]$ and $P \circ f=P f$, in which $P$ is a $q$-pseudo-differential operator $(q$-PDO) given by

$$
P=\sum_{i=-\infty}^{n} p_{i} \partial_{q}^{i}
$$

$[P f]$ denotes $P$ acting on the function $f$, while $P f$ indicates the multiplication of $P$ and $f$; that is, $\partial_{q} f=\theta(f) \partial_{q}+\left[\partial_{q} f\right]$.

It can be easily shown from (1) that when $q \rightarrow 1, \partial_{q}$ reduces to the ordinary differential operator $\partial_{x}$ and that $\theta$ and $\partial_{q}$ do not commute but satisfy

$$
\left[\partial_{q} \theta^{k}(f)\right]=q^{k} \theta^{k}\left[\partial_{q} f\right], \quad k \in Z
$$

Let $\partial_{q}^{-1}$ be the formal inverse of $\partial_{q}$ such as $\partial_{q} \partial_{q}^{-1} f=\partial_{q}^{-1} \partial_{q} f=$ $f$. In general, the $q$-deformed Leibnitz rule holds

$$
\partial_{q}^{n} f=\sum_{k \geq 0}\left(\begin{array}{l}
n \\
k
\end{array}\right)_{q} \theta^{n-k}\left(\partial_{q}^{k} f\right) \partial_{q}^{n-k}, \quad n \in Z,
$$

where $q$-number and $q$-binomial are defined by

$$
\begin{aligned}
& (n)_{q}=\frac{q^{n}-1}{q-1} \\
& \left(\begin{array}{l}
n \\
k
\end{array}\right)_{q}=\frac{(n)_{q}(n-1)_{q} \cdots(n-k+1)_{q}}{(1)_{q}(2)_{q} \cdots(k)_{q}} \\
& \left(\begin{array}{l}
n \\
0
\end{array}\right)_{q}=1 .
\end{aligned}
$$

For a $q$-PDO $P=\sum_{i=-\infty}^{n} p_{i} \partial_{q}^{i}$, we separate $P$ into the differential part $P_{+}=\sum_{i=0}^{n} p_{i} \partial_{q}^{i}$ and the integral part $P_{-}=$ $\sum_{i \leq-1} p_{i} \partial_{q}^{i}$. The conjugate operation $P^{*}$ is given by

$$
P^{*}=\sum_{i=-\infty}^{n}\left(\partial_{q}^{*}\right)^{i} p_{i}
$$

where $\partial_{q}^{*}=-\partial_{q} \theta^{-1}=-(1 / q) \partial_{1 / q},\left(\partial_{q}^{-1}\right)^{*}=\left(\partial_{q}^{*}\right)^{-1}=-\theta \partial_{q}^{-1}$.

The $q$-exponential function $E_{q}(x)$ is defined as

$$
E_{q}(x)=\exp \left(\sum_{k=1}^{\infty} \frac{(1-q)^{k}}{k\left(1-q^{k}\right)} x^{k}\right),
$$

satisfying $\left[\partial_{q}^{k} E_{q}(x z)\right]=z^{k} E_{q}(x z), k \in Z$.
The extended $q-\mathrm{KPH}$ was given by [16]

$$
\begin{aligned}
L_{t_{n}} & =\left[B_{n}, L\right], \quad B_{n}=\left(L^{n}\right)_{\geq 0}, \\
L_{\tau_{k}} & =\left[B_{k}+\sum_{j=1}^{m} \phi_{j} \partial_{q}^{-1} \psi_{j}, L\right], \quad B_{k}=\left(L^{k}\right)_{\geq 0}, k \neq n, \\
\phi_{j, t_{n}} & =\left[B_{n} \phi_{j}\right], \\
\psi_{j, t_{n}} & =-\left[B_{n}^{*} \psi_{j}\right],
\end{aligned}
$$

$$
j=1, \ldots, m
$$

where $L=\partial_{q}+u_{0}+u_{1} \partial_{q}^{-1}+u_{2} \partial_{q}^{-2}+\cdots$ and the coefficients $u_{i}(i=0,1, \ldots)$ are the functions of $t=\left(x, t_{1}, \ldots\right)$.

The commutativity of (9a), (9b), and (9c) leads to the zero-curvature representation of $q-\mathrm{KPH}((9 \mathrm{a}),(9 \mathrm{~b})$, and (9c)). As the $n$-reduction of the extended $q-\mathrm{KPH}$, the $q$ NKdVHSCS is defined as follows [16]:

$$
\begin{aligned}
B_{n, \tau_{k}} & =\left[B_{k}+\sum_{j=1}^{m} \phi_{j} \partial_{q}^{-1} \psi_{j}, B_{n}\right], \quad B_{k}=\left(B_{n}\right)_{\geq 0}^{k / n} \\
{\left[B_{n} \phi_{j}\right] } & =\lambda_{j}^{n} \phi_{j}, \\
{\left[B_{n}^{*} \psi_{j}\right] } & =\mu_{j}^{n} \psi_{j}, \quad j=1, \ldots, m .
\end{aligned}
$$

Under (10b) and (10c), the Lax representation for (10a) is

$$
\left[B_{n} \varphi\right]=\lambda \varphi,
$$

$$
\varphi_{\tau_{k}}=\left[\left(B_{k}+\sum_{j=1}^{m} \phi_{j} \partial_{q}^{-1} \psi_{j}\right) \varphi\right] .
$$

We find that when $m=0, q$-NKdVHSCS ((10a), (10b), and (10c) and (11a) and (11b)) can be reduced to the $q-\mathrm{NKdVH}$ and its related Lax representation, respectively. In addition, when $n=2, k=1$ ((10a), (10b), and (10c)) becomes the first nontrivial soliton equation of $q$-KdVHSCS given by

$$
\begin{gathered}
v_{1, \tau_{1}}+e_{1}+\sum_{j=1}^{m} g_{j 1}=0 \\
v_{0, \tau_{3}}+e_{0}+\sum_{j=1}^{m} g_{j 0}=0 \\
\theta\left(v_{1}\right)-v_{1}+u_{0}-\theta^{2}\left(u_{0}\right)=0, \\
{\left[\left(\partial_{q}^{2}+v_{1} \partial_{q}+v_{0}\right) \varphi_{j}\right]-\lambda_{j}^{2} \varphi_{j}=0,} \\
{\left[\left(\partial_{q}^{2}+v_{1} \partial_{q}+v_{0}\right)^{*} \psi_{j}\right]-\mu_{j}^{2} \psi_{j}=0, \quad j=1, \ldots, m .}
\end{gathered}
$$

\section{The Auto Darboux-Bäcklund Transformation (DBT) for $q$-NKdVHSCS}

In this section, we will focus on the construction of auto DBT for $q$-NKdVHSCS. 
Theorem 1. Assume $B_{n}, \phi_{j}, \psi_{j}(j=1, \ldots, m)$ be the solution of q-NKdVHSCS ((10a), (10b), and (10c)), and $h_{1}$ satisfies (11a) and (11b) with $\lambda=\lambda_{1}^{n}$; the DBT is defined by

$$
\begin{aligned}
& \bar{B}_{n}=T_{1} B_{n} T_{1}^{-1}=\partial_{q}^{n}+\bar{v}_{n-1} \partial_{q}^{n-1}+\cdots+\bar{v}_{1} \partial_{q}+\bar{v}_{0}, \\
& \bar{\phi}=\left[T_{1} \phi\right]=\frac{W_{q}\left[h_{1}, \phi\right]}{h_{1}}, \\
& \bar{\phi}_{j}=\left[T_{1} \phi_{j}\right]=\frac{W_{q}\left[h_{1}, \phi_{j}\right]}{h_{1}}, \\
& \bar{\psi}_{j}=\left[\left(T_{1}^{-1}\right)^{*} \psi_{j}\right]=-\frac{\theta\left(\Omega\left(h_{1}, \psi_{j}\right)\right)}{\theta\left(h_{1}\right)}, \quad \\
& \quad j=1, \ldots, m,
\end{aligned}
$$

Then $\bar{B}_{n}, \bar{\phi}, \bar{\phi}_{j}, \bar{\psi}_{j}(j=1, \ldots, m)$ satisfy (10b) and (10c) and (11a) and (11b) and hence are the solution of q-NKdVHSCS ((10a), (10b), and (10c)), where $T_{1}=\theta\left(h_{1}\right) \partial_{q} h_{1}^{-1}=\partial_{q}-\alpha_{1}$, $\alpha_{1}=\left[\partial_{q} h_{1}\right] / h_{1}$, and $W_{q}\left[h_{1}, \phi_{j}\right]$ and $\Omega_{q}\left[h_{1}, \psi_{j}\right]$ are defined as follows:

$$
\begin{aligned}
& W_{q}\left[\phi_{1}, \phi\right]=\left|\begin{array}{cc}
h_{1} & \phi \\
{\left[\partial_{q} h_{1}\right]} & {\left[\partial_{q} \phi\right]}
\end{array}\right|, \\
& \Omega\left(h_{1}, \psi_{j}\right)=\left[\partial_{q}^{-1} h_{1} \psi_{j}\right] .
\end{aligned}
$$

Remark 2. Here it should be pointed out that the formula holds

$$
\begin{aligned}
& \bar{B}_{k}=T_{1} B_{k} T_{1}^{-1}+T_{1, \tau_{k}} T_{1}^{-1}, \\
& B_{k}=\left(B_{n}\right)_{\geq 0}^{k / n}, \\
& \bar{B}_{k}=\left(\bar{B}_{n}\right)_{\geq 0}^{k / n},
\end{aligned}
$$

where the gauge operator $T_{1}$ is defined above. The proof has been given in [7].

Proof. (1) We firstly show that $\bar{B}_{n}, \bar{\phi}_{j}, \bar{\psi}_{j}(j=1, \ldots, m)$ satisfy (10b) and (10c).

Noting that $B_{n}, \phi_{j}, \psi_{j}(j=1, \ldots, m)$ are the solution of (10a), (10b), and (10c), we have

$$
\begin{aligned}
& {\left[B_{n} \phi_{j}\right]=\lambda_{j}^{n} \phi_{j},} \\
& {\left[B_{n}^{*} \psi_{j}\right]=\mu_{j}^{n} \psi_{j} .}
\end{aligned}
$$

Hence,

$$
\begin{aligned}
{\left[\bar{B}_{n} \bar{\phi}_{j}\right]-\lambda_{j}^{n} \bar{\phi}_{j}=} & {\left[T_{1} B_{n} T_{1}^{-1}\left[T_{1} \phi_{j}\right]\right]-\lambda_{j}^{n} \bar{\phi}_{j} } \\
= & {\left[T_{1}\left[B_{n} \phi_{j}\right]\right]-\lambda_{j}^{n} \bar{\phi}_{j} } \\
= & \lambda_{j}^{n}\left(\left[T_{1} \phi_{j}\right]-\bar{\phi}_{j}\right)=0, \\
{\left[\bar{B}_{n}^{*} \bar{\psi}_{j}\right]-\mu_{j}^{n} \bar{\psi}_{j}=} & {\left[\left(T_{1}^{-1}\right)^{*} B_{n}^{*} T_{1}^{*}\left[\left(T_{1}^{-1}\right)^{*} \psi_{j}\right]\right] } \\
& -\mu_{j}^{n} \bar{\psi}_{j}=\left[\left(T_{1}^{-1}\right)^{*} B_{n}^{*} \psi_{j}\right]-\mu_{j}^{n} \bar{\psi}_{j} \\
= & 0 .
\end{aligned}
$$

(2) We finally show that $\bar{B}_{n}, \bar{\phi}, \bar{\phi}_{j}, \bar{\psi}_{j}$ satisfy (11a) and (11b). Since the proof of (11a) is the same as the case (1), we only need to verify that $\bar{B}_{n}, \bar{\phi}, \bar{\phi}_{j}, \bar{\psi}_{j}$ satisfy (11b); that is,

$$
\bar{\phi}_{\tau_{k}}-\left[\left(\bar{B}_{k}+\sum_{j=1}^{m} \bar{\phi}_{j} \partial_{q}^{-1} \bar{\psi}_{j}\right) \bar{\phi}\right]=0, \quad \bar{B}_{k}=\left(\bar{B}_{n}\right)_{\geq 0}^{k / n} .
$$

Noting that $\bar{\phi}_{\tau_{k}}=\left[T_{1} \phi\right]_{\tau_{k}}=\left[T_{1, \tau_{k}} \phi\right]+\left[T_{1} \phi_{\tau_{k}}\right]$ and $\phi_{\tau_{k}}=$ $\left[\left(B_{k}+\sum_{j=1}^{m} \phi_{j} \partial_{q}^{-1} \psi_{j}\right) \phi\right]$, we get

$$
\begin{gathered}
\bar{\phi}_{\tau_{k}}-\left[\left(\bar{B}_{k}+\sum_{j=1}^{m} \bar{\phi}_{j} \partial_{q}^{-1} \bar{\psi}_{j}\right) \bar{\phi}\right]=\left[T_{1, \tau_{k}} \phi\right]+\left[T_{1} \phi_{\tau_{k}}\right] \\
-\left[\left(\bar{B}_{k}+\sum_{j=1}^{m} \bar{\phi}_{j} \partial_{q}^{-1} \bar{\psi}_{j}\right)\left[T_{1} \phi\right]\right]=\left[\left(T_{1, \tau_{k}}+T_{1} B_{k}\right.\right. \\
\left.\left.-\bar{B}_{k} T_{1}+\sum_{j=1}^{m} T_{1} \phi_{j} \partial_{q}^{-1} \psi_{j}-\sum_{j=1}^{m} \bar{\phi}_{j} \partial_{q}^{-1} \bar{\psi}_{j} T_{1}\right) \phi\right] .
\end{gathered}
$$

According to Remark 2, we have

$$
T_{1, \tau_{k}}+T_{1} B_{k}-\bar{B}_{k} T_{1}=0 .
$$

Next, we prove

$$
\sum_{j=1}^{m}\left(T_{1} \phi_{j} \partial_{q}^{-1} \psi_{j}-\bar{\phi}_{j} \partial_{q}^{-1} \bar{\psi}_{j} T_{1}\right)=0
$$

Since $T_{1}=\partial_{q}-\alpha_{1}$ and $\partial_{q} \phi_{j}=\theta\left(\phi_{j}\right) \partial_{q}+\left[\partial_{q} \phi_{j}\right]$, then $\forall j$, we obtain by the tedious computation

$$
\begin{aligned}
& T_{1} \phi_{j} \partial_{q}^{-1} \psi_{j}-\bar{\phi}_{j} \partial_{q}^{-1} \bar{\psi}_{j} T_{1} \\
& \quad=\left(\partial_{q}-\alpha_{1}\right) \phi_{j} \partial_{q}^{-1} \psi_{j}-\bar{\phi}_{j} \partial_{q}^{-1} \bar{\psi}_{j} \partial_{q}+\bar{\phi}_{j} \partial_{q}^{-1} \bar{\psi}_{j} \alpha_{1} \\
& \quad=\theta\left(\phi_{j}\right) \psi_{j}+\bar{\phi}_{j} \frac{\Omega\left(h_{1}, \psi_{j}\right)}{h_{1}} .
\end{aligned}
$$


In addition, we also have

$$
\begin{aligned}
\theta\left(\phi_{j}\right) \psi_{j}+\bar{\phi}_{j} \frac{\Omega\left(h_{1}, \psi_{j}\right)}{h_{1}} & =\frac{\left[\theta\left(\phi_{j}\right) \partial_{q} \Omega\left(\phi_{1}, \psi_{j}\right)\right]+\left[T_{1} h_{1}\right] \Omega\left(h_{1}, \psi_{j}\right)}{h_{1}} \\
& =\frac{\left[\partial_{q} \phi_{j} \Omega\left(h_{1}, \psi_{j}\right)\right]-\left[\partial_{q} \phi_{j}\right] \Omega\left(h_{1}, \psi_{j}\right)+\left(\left[\partial_{q} \phi_{j}\right]-\alpha_{1} \phi_{j}\right) \Omega\left(h_{1}, \psi_{j}\right)}{h_{1}} \\
& =\frac{\left[\left(\partial_{q}-\alpha_{1}\right) \phi_{j} \Omega\left(h_{1}, \psi_{j}\right)\right]}{h_{1}}=\frac{\left[T_{1}\left[\left(\phi_{j} \partial_{q}^{-1} \psi_{j}\right) h_{1}\right]\right]}{h_{1}} .
\end{aligned}
$$

Substituting (22b) into (22a) leads to

$$
\begin{aligned}
\sum_{j=1}^{m} & \left(T_{1} \phi_{j} \partial_{q}^{-1} \psi_{j}-\bar{\phi}_{j} \partial_{q}^{-1} \bar{\psi}_{j} T_{1}\right) \\
& =\frac{1}{h_{1}}\left[T_{1}\left[\sum_{j=1}^{m}\left(\phi_{j} \partial_{q}^{-1} \psi_{j}\right) h_{1}\right]\right] .
\end{aligned}
$$

Since $h_{1}$ is the solution of (11a) and (11b) with $\lambda=\lambda_{1}^{n}$, we have

$$
\begin{aligned}
h_{1, \tau_{k}} & =\left[\left(B_{k}+\sum_{j=1}^{m} \phi_{j} \partial_{q}^{-1} \psi_{j}\right) h_{1}\right] \Longrightarrow \\
{\left[\left(\sum_{j=1}^{m} \phi_{j} \partial_{q}^{-1} \psi_{j}\right) h_{1}\right] } & =h_{1, \tau_{k}}-\left[B_{k} h_{1}\right] .
\end{aligned}
$$

Moreover, by the property of determinant, we have

$$
\left[T_{1} h_{1}\right]=\frac{W_{q}\left[h_{1}, h_{1}\right]}{h_{1}}=0 .
$$

Differentiating both sides of (25) with respect to $\tau_{k}$ yields

$$
\begin{aligned}
{\left[T_{1, \tau_{k}} \phi_{1}\right]+\left[T_{1} \phi_{1, \tau_{k}}\right] } & =0 \Longrightarrow \\
{\left[T_{1} \phi_{1, \tau_{k}}\right] } & =-\left[T_{1, \tau_{k}} \phi_{1}\right] .
\end{aligned}
$$

From (23), (24), and (26), we have

$$
\begin{aligned}
& \sum_{j=1}^{m}\left(T_{1} \phi_{j} \partial_{q}^{-1} \psi_{j}-\bar{\phi}_{j} \partial_{q}^{-1} \bar{\psi}_{j} T_{1}\right) \\
& \quad=-\frac{1}{\phi_{1}}\left[\left(T_{1, \tau_{k}}+T_{1} B_{k}\right) \phi_{1}\right]=-\frac{1}{\phi_{1}}\left[\bar{B}_{k}\left[T_{1} \phi_{1}\right]\right] \\
& \quad=0 .
\end{aligned}
$$

This completes the proof.

Obviously, Theorem 1 provides an auto DBT for $q$ NKdVHSCS ((10a), (10b), and (10c)). However, this DBT does not enable us to obtain the new solution of $q$-NKdVHSCS ((10a), (10b), and (10c)). So we have to seek for nonauto DBTs between the two $q$-NKdVHSCS ((10a), (10b), and (10c)) with different degrees of sources.

\section{The Nonauto DBTs of $q$-NKdVHSCS}

In this section, we will construct the nonauto DBTs of $q$ NKdVHSCS ((10a), (10b), and (10c)), which enables us to obtain the new solution of $q$-NKdVHSCS from the known solution of $q-\mathrm{NKdVH}$.

Theorem 3. Given $B_{n}, \phi_{j}, \psi_{j}(j=1, \ldots, m)$ the solution for $q$-NKdVHSCS ((10a), (10b), and (10c)), let $f_{1}, g_{1} \equiv \phi_{m+1}$ be two independent eigenfunctions of (11a) and (11b) with $\lambda=$ $\lambda_{m+1}^{n}$. Let $b_{1}\left(\tau_{k}\right)$ be a function of $\tau_{k}$ such that $b_{1}\left(\tau_{k}\right)_{\tau_{k}}=$ $(-1)^{m+1} \beta_{1}\left(\tau_{k}\right) \eta_{1}\left(\tau_{k}\right)$. Denote $h_{1}=f_{1}+b_{1}\left(\tau_{k}\right) g_{1}$.

The DBT is defined by

$$
\begin{aligned}
\bar{B}_{n} & =T_{1} B_{n} T_{1}^{-1}=\partial_{q}^{n}+\bar{v}_{n-1} \partial_{q}^{n-1}+\cdots+\bar{v}_{1} \partial_{q}+\bar{v}_{0}, \\
\bar{\phi} & =\left[T_{1} \phi\right]=\frac{W_{q}\left[h_{1}, \phi\right]}{h_{1}}, \\
\bar{\phi}_{j} & =\left[T_{1} \phi_{j}\right]=\frac{W_{q}\left[h_{1}, \phi_{j}\right]}{h_{1}}, \\
\bar{\psi}_{j} & =\left[\left(T_{1}^{-1}\right)^{*} \psi_{j}\right]=-\frac{\theta\left(\Omega\left(h_{1}, \psi_{j}\right)\right)}{\theta\left(h_{1}\right)}, \\
\bar{\phi}_{m+1} & =-\beta_{1}\left(\tau_{k}\right)\left[T_{1} g_{1}\right], \\
\bar{\psi}_{m+1} & =(-1)^{m+1} \eta_{1}\left(\tau_{k}\right) \frac{1}{\theta\left(h_{1}\right)},
\end{aligned}
$$

where $T_{1}=\partial_{q}-\alpha_{1}, \alpha_{1}=\left[\partial_{q} h_{1}\right] / h_{1}$, and then $\bar{B}_{n}, \bar{\phi}, \bar{\phi}_{j}, \bar{\psi}_{j}(j=$ $1, \ldots, m), \bar{\phi}_{m+1}, \bar{\psi}_{m+1}$ satisfy (10b) and (11a) and (11b) with $m$ replaced by $m+1$; hence $\bar{B}_{n}, \bar{\phi}_{j}, \bar{\psi}_{j}(j=1, \ldots, m), \bar{\phi}_{m+1}, \bar{\psi}_{m+1}$ are the solution of $q-N K d V H S C S$ ((10a), (10b), and (10c)) with $m$ replaced by $m+1$.

Proof. (1) We firstly show that $\bar{B}_{n}, \bar{\phi}, \bar{\phi}_{j}, \bar{\psi}_{j}(j=1, \ldots, m)$, $\bar{\phi}_{m+1}, \bar{\psi}_{m+1}$ are the solution of (10b) and (10c).

With the same proof as Theorem $1, \bar{B}_{n}, \bar{\phi}, \bar{\phi}_{j}, \bar{\psi}_{j}(j=$ $1, \ldots, m)$ can be shown to be the solution of (10b) and (10c). 
Here we only need to show that $\bar{\phi}_{m+1}, \bar{\psi}_{m+1}$ are also the solution of (10b) and (10c). Consider

$$
\begin{aligned}
{\left[\bar{B}_{n} \bar{\phi}_{m+1}\right] } & =-\beta_{1}\left(\tau_{k}\right)\left[T_{1} B_{n} T_{1}^{-1}\left[T_{1} g_{1}\right]\right] \\
& =-\beta_{1}\left(\tau_{k}\right)\left[T_{1}\left[B_{n} g_{1}\right]\right] \\
& =-\lambda_{m+1}^{n} \beta_{1}\left(\tau_{k}\right)\left[T_{1} g_{1}\right]=\lambda_{m+1}^{n} \bar{\phi}_{m+1} .
\end{aligned}
$$

Taking a proper solution $\psi_{m+1}$ of (10c) with $\mu=\mu_{m+1}^{n}$ such that $\Omega\left(h_{1}, \psi_{m+1}\right)=-1$, then we get

$$
\begin{aligned}
& {\left[\bar{B}_{n}^{*} \bar{\psi}_{m+1}\right]=(-1)^{m+1} \eta_{1}\left(\tau_{k}\right)} \\
& \cdot\left[\left(T_{1}^{-1}\right)^{*} B_{n}^{*} T_{1}^{*}\left(\frac{1}{\theta\left(h_{1}\right)}\right)\right]=(-1)^{m+1} \eta_{1}\left(\tau_{k}\right) \\
& \cdot\left[\left(T_{1}^{-1}\right)^{*} B_{n}^{*} T_{1}^{*}\left(-\frac{\Omega\left(h_{1}, \psi_{m+1}\right)}{\theta\left(h_{1}\right)}\right)\right] .
\end{aligned}
$$

Noting that $\left[\left(T_{1}^{-1}\right)^{*} \psi_{m+1}\right]=-\left(\Omega\left(h_{1}, \psi_{m+1}\right)\right) / \theta\left(h_{1}\right)$, we derive from (30)

$$
\begin{aligned}
& {\left[\bar{B}_{n}^{*} \bar{\phi}_{m+1}\right]} \\
& \quad=(-1)^{m+1} \eta_{1}\left(\tau_{k}\right)\left[\left(T_{1}^{-1}\right)^{*} B_{n}^{*} T_{1}^{*}\left[\left(T_{1}^{-1}\right)^{*} \psi_{m+1}\right]\right] \\
& \quad=(-1)^{m+1} \eta_{1}\left(\tau_{k}\right)\left[\left(T_{1}^{-1}\right)^{*}\left[B_{n}^{*} \psi_{m+1}\right]\right] \\
& \quad=\mu_{m+1}^{n} \bar{\psi}_{m+1} .
\end{aligned}
$$

(2) We finally show that $\bar{B}_{n}, \bar{\phi}_{j}, \bar{\psi}_{j}(j=1, \ldots, m), \bar{\phi}_{m+1}$, $\bar{\psi}_{m+1}$ are the solution of (11a) and (11b) with $m$ replaced by $m+$ 1. Evidently we only need to prove $\bar{B}_{n}, \bar{\phi}_{j}, \bar{\psi}_{j}(j=1, \ldots, m)$, $\bar{\phi}_{m+1}, \bar{\psi}_{m+1}$ satisfy (11b); that is, $\bar{\phi}_{\tau_{k}}-\left[\left(\bar{B}_{k}+\sum_{j=1}^{m+1} \bar{\phi}_{j} \partial_{q}^{-1} \bar{\psi}_{j}\right) \bar{\phi}\right]=$ 0 .

Noting that $\bar{\phi}=\left[T_{1} \phi\right] \Rightarrow \bar{\phi}_{\tau_{k}}=\left[T_{1} \phi\right]_{\tau_{k}}=\left[T_{1, \tau_{k}} \phi\right]+$ $\left[T_{1} \phi_{\tau_{k}}\right]$, we have

$$
\begin{gathered}
\bar{\phi}_{\tau_{k}}-\left[\left(\bar{B}_{k}+\sum_{j=1}^{m+1} \bar{\phi}_{j} \partial_{q}^{-1} \bar{\psi}_{j}\right) \bar{\phi}\right]=\left[\left(T_{1, \tau_{k}}+T_{1} B_{k}\right.\right. \\
\left.\left.-\bar{B}_{k} T_{1}+\sum_{j=1}^{m} T_{1} \phi_{j} \partial_{q}^{-1} \psi_{j}-\sum_{j=1}^{m+1} \bar{\phi}_{j} \partial_{q}^{-1} \bar{\psi}_{j} T_{1}\right) \phi\right] .
\end{gathered}
$$

From (15), a direct computation leads to

$$
T_{1, \tau_{k}}+T_{1} B_{k}-\bar{B}_{k} T_{1}+\frac{b_{1}\left(\tau_{k}\right)_{\tau_{k}}\left[T_{1} g_{1}\right]}{h_{1}}=0 .
$$

Noticing that

$$
\begin{aligned}
& \sum_{j=1}^{m} T_{1} \phi_{j} \partial_{q}^{-1} \psi_{j}-\sum_{j=1}^{m+1} \bar{\phi}_{j} \partial_{q}^{-1} \bar{\psi}_{j} T_{1} \\
& =\sum_{j=1}^{m}\left(T_{1} \phi_{j} \partial_{q}^{-1} \psi_{j}-\bar{\phi}_{j} \partial_{q}^{-1} \bar{\psi}_{j} T_{1}\right) \\
& -\bar{\phi}_{m+1} \partial_{q}^{-1} \bar{\psi}_{m+1} T_{1},
\end{aligned}
$$

then $\forall j=1, \ldots, m$, we obtain by the tedious computation

$$
\begin{aligned}
& T_{1} \phi_{j} \partial_{q}^{-1} \psi_{j}-\bar{\phi}_{j} \partial_{q}^{-1} \bar{\psi}_{j} T_{1} \\
& \quad=\theta\left(\phi_{j}\right) \psi_{j}+\bar{\phi}_{j} \frac{\theta\left(\Omega\left(h_{1}, \psi_{j}\right)\right)}{\theta\left(h_{1}\right)} .
\end{aligned}
$$

Substituting (33b) into (33a), we get

$$
\begin{aligned}
\sum_{j=1}^{m} T_{1} \phi_{j} \partial_{q}^{-1} \psi_{j}-\sum_{j=1}^{m+1} \bar{\phi}_{j} \partial_{q}^{-1} \bar{\psi}_{j} T_{1} \\
=\sum_{j=1}^{m}\left(\theta\left(\phi_{j}\right) \psi_{j}+\bar{\phi}_{j} \frac{\theta\left(\Omega\left(h_{1}, \psi_{j}\right)\right)}{\theta\left(h_{1}\right)}\right) \\
\quad-\bar{\phi}_{m+1} \partial_{q}^{-1} \bar{\psi}_{m+1} \partial_{q}+\bar{\phi}_{m+1} \partial_{q}^{-1} \bar{\psi}_{m+1} \alpha_{1} \\
=\sum_{j=1}^{m}\left(\theta\left(\phi_{j}\right) \psi_{j}+\bar{\phi}_{j} \frac{\theta\left(\Omega\left(h_{1}, \psi_{j}\right)\right)}{\theta\left(h_{1}\right)}\right) \\
\quad-(-1)^{m+1} \eta_{1}\left(\tau_{k}\right) \bar{\phi}_{m+1} \partial_{q}^{-1}\left(\partial_{q} \frac{1}{h_{1}}-\left[\partial_{q} \frac{1}{h_{1}}\right]\right) \\
\quad+\bar{\phi}_{m+1} \partial_{q}^{-1} \bar{\psi}_{m+1} \alpha_{1} \\
=\sum_{j=1}^{m}\left(\theta\left(\phi_{j}\right) \psi_{j}+\bar{\phi}_{j} \frac{\theta\left(\Omega\left(h_{1}, \psi_{j}\right)\right)}{\theta\left(h_{1}\right)}\right) \\
+(-1)^{m+1} \frac{\beta_{1}\left(\tau_{k}\right) \eta_{1}\left(\tau_{k}\right)\left[T_{1} g_{1}\right]}{h_{1}} .
\end{aligned}
$$

In addition, since $f_{1}, g_{1}$ are the solutions of (11a) and (11b), we have

$$
\begin{aligned}
& f_{1, \tau_{k}}=\left[\left(B_{k}+\sum_{j=1}^{m} \phi_{j} \partial_{q}^{-1} \psi_{j}\right) f_{1}\right], \\
& g_{1, \tau_{k}}=\left[\left(B_{k}+\sum_{j=1}^{m} \phi_{j} \partial_{q}^{-1} \psi_{j}\right) g_{1}\right] ;
\end{aligned}
$$

hence

$$
\begin{aligned}
h_{1, \tau_{k}}= & f_{1, \tau_{k}}+b_{1}\left(\tau_{k}\right) g_{1, \tau_{k}} \\
& +b_{1}\left(\tau_{k}\right)_{\tau_{k}} g_{1} \\
= & {\left[\left(B_{k}+\sum_{j=1}^{m} \phi_{j} \partial_{q}^{-1} \psi_{j}\right) h_{1}\right] } \\
& +b_{1}\left(\tau_{k}\right)_{\tau_{k}} g_{1} \Longrightarrow \\
{\left[\sum_{j=1}^{m}\left(\phi_{j} \partial_{q}^{-1} \psi_{j}\right) h_{1}\right]=} & h_{1, \tau_{k}}-\left[B_{k} h_{1}\right]-b_{1}\left(\tau_{k}\right)_{\tau_{k}} g_{1} .
\end{aligned}
$$


Noting $\left[T_{1} h_{1}\right]=W_{q}\left[h_{1}, h_{1}\right] / h_{1}=0$ and differentiating both sides of this equation with respect to $\tau_{k}$ lead to

$$
\begin{aligned}
{\left[T_{1, \tau_{k}} h_{1}\right]+\left[T_{1} h_{1, \tau_{k}}\right] } & =0 \Longrightarrow \\
{\left[T_{1} h_{1, \tau_{k}}\right] } & =-\left[T_{1, \tau_{k}} h_{1}\right] .
\end{aligned}
$$

Rewriting (33c) leads to

$$
\begin{aligned}
\sum_{j=1}^{m} T_{1} \phi_{j} \partial_{q}^{-1} \psi_{j}-\sum_{j=1}^{m+1} \bar{\phi}_{j} \partial_{q}^{-1} \bar{\psi}_{j} T_{1} \\
=\frac{\sum_{j=1}^{m}\left[T_{1}\left(\phi_{j} \partial_{q}^{-1} \psi_{j}\right) h_{1}\right]}{h_{1}} \\
+(-1)^{m+1} \frac{\beta_{1}\left(\tau_{k}\right) \eta_{1}\left(\tau_{k}\right)\left[T_{1} g_{1}\right]}{h_{1}} .
\end{aligned}
$$

Combining (32a) and (32b) and (35) and (36), we get

$$
\begin{gathered}
\frac{\sum_{j=1}^{m}\left[T_{1}\left(\phi_{j} \partial_{q}^{-1} \psi_{j}\right) h_{1}\right]}{h_{1}}=\frac{T_{1}\left[\sum_{j=1}^{m}\left(\phi_{j} \partial_{q}^{-1} \psi_{j}\right) h_{1}\right]}{h_{1}} \\
=\frac{\left[T_{1} h_{1, \tau_{k}}\right]-\left[T_{1} B_{k} h_{1}\right]-b_{1}\left(\tau_{k}\right)_{\tau_{k}}\left[T_{1} g_{1}\right]}{h_{1}} \\
=-\frac{\left[T_{1, \tau_{k}} h_{1}\right]+\left[T_{1} B_{k} h_{1}\right]}{h_{1}}-\frac{b_{1}\left(\tau_{k}\right)_{\tau_{k}}\left[T_{1} g_{1}\right]}{h_{1}} \\
=-\frac{\left[B_{k}\left[T_{1} h_{1}\right]\right]}{h_{1}}+\frac{b_{1}\left(\tau_{k}\right)_{\tau_{k}}\left[T_{1} g_{1}\right]}{h_{1}} \\
-\frac{b_{1}\left(\tau_{k}\right)_{\tau_{k}}\left[T_{1} g_{1}\right]}{h_{1}}=-\frac{\left[B_{k}\left[T_{1} h_{1}\right]\right]}{h_{1}}=0 .
\end{gathered}
$$

Noting $b_{1}\left(\tau_{k}\right)_{\tau_{k}}=(-1)^{m+1} \beta_{1}\left(\tau_{k}\right) \eta_{1}\left(\tau_{k}\right)$, we immediately get from $(38)$

$$
\bar{\phi}_{\tau_{k}}-\left[\left(\bar{B}_{k}+\sum_{j=1}^{m+1} \bar{\phi}_{j} \partial_{q}^{-1} \bar{\psi}_{j}\right) \bar{\phi}\right]=0 .
$$

This completes the proof.

Theorem 4 (the $N$-times repeated nonauto DBT). Given $B_{n}, \phi_{1}, \ldots, \phi_{m}, \psi_{1} \ldots, \psi_{m}$ are the solution for q-NKdVHSCS ((10a), (10b), and (10c)), $f_{1}, \ldots, f_{N}, g_{1}, \ldots, g_{N}$ are independent eigenfunctions of (11a) and (11b) with $\lambda=$ $\lambda_{m+1}^{n}, \ldots, \lambda_{m+N}^{n} \cdot b_{i}\left(\tau_{k}\right), i=1, \ldots, N$, are functions of $\tau_{k}$ such that $b_{i}\left(\tau_{k}\right)_{\tau_{k}}=(-1)^{m+N} \beta_{i}\left(\tau_{k}\right) \eta_{i}\left(\tau_{k}\right)$.

Denote $h_{i}=f_{i}+b_{i}\left(\tau_{k}\right) g_{i}$. The $N$-times repeated DBT is defined by

$$
\begin{aligned}
& B_{n}^{(N)}=T_{N} B_{n} T_{N}^{-1}=\partial_{q}^{n}+v_{n-1}^{(N)} \partial_{q}^{n-1}+\cdots+v_{1}^{(N)} \partial_{q} \\
& +v_{0}^{(N)}, \\
& \phi^{(N)}=\left[T_{N} \phi\right]=\frac{W_{q}\left[h_{1}, h_{2}, \ldots, h_{N}, \phi\right]}{W_{q}\left[h_{1}, h_{2}, \ldots, h_{N}\right]}, \\
& \phi_{j}^{(N)}=\left[T_{N} \phi_{j}\right]=\frac{W_{q}\left[h_{1}, h_{2}, \ldots, h_{N}, \phi_{j}\right]}{W_{q}\left[h_{1}, h_{2}, \ldots, h_{N}\right]}, \\
& \psi_{j}^{(N)}=\left[\left(T_{N}^{-1}\right)^{*} \psi_{j}\right]=-\frac{\theta\left(G_{q}\left[h_{1}, h_{2}, \ldots, h_{N}, \psi_{j}\right]\right)}{\theta\left(W_{q}\left[h_{1}, h_{2}, \ldots, h_{N}\right]\right)}, \\
& \phi_{m+i}^{(N)}=-\beta_{i}\left(\tau_{s}\right)\left[T_{N} g_{i}\right], \\
& \psi_{m+i}^{(N)}=(-1)^{m+i} \eta_{i}\left(\tau_{k}\right) \\
& \quad \cdot \frac{\theta\left(W_{q}\left[h_{1}, \ldots, h_{i-1}, h_{i+1}, \ldots, h_{N}\right]\right)}{\theta\left(W_{q}\left[h_{1}, h_{2}, \ldots, h_{N}\right]\right)},
\end{aligned}
$$

where

$$
\begin{aligned}
& T_{N}=\frac{1}{W_{q}\left[h_{1}, h_{2}, \ldots, h_{N}\right]} \\
& \cdot\left[\begin{array}{ccccc}
h_{1} & h_{2} & \cdots & h_{N} & 1 \\
{\left[\partial_{q} h_{1}\right]} & {\left[\partial_{q} h_{2}\right]} & \cdots & {\left[\partial_{q} h_{N}\right]} & \partial_{q} \\
\vdots & \vdots & \vdots & \vdots \\
{\left[\partial_{q}^{N} h_{1}\right]} & {\left[\partial_{q}^{N} h_{2}\right]} & \cdots & {\left[\partial_{q}^{N} h_{N}\right]} & \partial_{q}^{N}
\end{array}\right], \\
& G_{q}\left[h_{1}, h_{2}, \ldots, h_{N}\right] \\
& =\left|\begin{array}{cccc}
h_{1} & h_{2} & \cdots & h_{N} \\
\vdots & \vdots & \vdots & \vdots \\
{\left[\partial_{q}^{N-2} h_{1}\right]} & {\left[\partial_{q}^{N-2} h_{2}\right]} & \cdots & {\left[\partial_{q}^{N-2} h_{N}\right]} \\
{\left[\partial_{q}^{-1} h_{1} \psi_{j}\right]} & {\left[\partial_{q}^{-1} h_{2} \psi_{j}\right]} & \cdots & {\left[\partial_{q}^{-1} h_{N} \psi_{j}\right]}
\end{array}\right|, \\
& T_{N}=D_{N} D_{N-1} \cdots D_{1} \text {, } \\
& D_{k}=\left(\partial_{q}-\alpha_{k}^{(k-1)}\right) \text {, }
\end{aligned}
$$




$$
\begin{aligned}
& \alpha_{i}^{(k)}=\frac{\left[\partial_{q} h_{i}^{(k)}\right]}{h_{i}^{(k)}}, \\
& h_{i}^{(k)}=\left[T_{k} h_{i}\right], \quad k=0,1, \ldots, N-1 ;
\end{aligned}
$$

then $B_{n}^{(N)}, \phi_{j}^{(N)}, \psi_{j}^{(N)},(j=1, \ldots, m)$ satisfy $(10 b)$ and $(10 c)$ and (11a) and (11b) with $m$ replaced by $m+N$; hence $B_{n}^{(N)}, \phi_{j}^{(N)}, \psi_{j}^{(N)},(j=1, \ldots, m), \phi_{m+i}^{(N)}, \psi_{m+i}^{(N)},(i=1, \ldots, N)$ are the solution of $q$-NKdVHSCS ((10a), (10b), and (10c)) with $m$ replaced by $m+N$.

Proof. With the same method as Theorem 3, we can show that $\phi_{j}^{(N)}, \psi_{j}^{(N)},(j=1, \ldots, m), \phi_{m+i}^{(N)}, \psi_{m+i}^{(N)},(i=1, \ldots, N)$ satisfy (10b), (10c), and (11a). Here we only need to show $B_{n}^{(N)}, \phi^{(N)}, \phi_{j}^{(N)}, \psi_{j}^{(N)},(j=1, \ldots, m), \phi_{m+i}^{(N)}, \psi_{m+i}^{(N)},(i=$ $1, \ldots, N)$ satisfy (11b). Next we will show it by the mathematical induction method. Theorem 3 indicates $B_{n}^{(N)}, \phi_{j}^{(N)}, \psi_{j}^{(N)},(j=1, \ldots, m), \phi_{m+i}^{(N)}, \psi_{m+i}^{(N)},(i=1, \ldots, N)$ satisfy (11b) in the case of $N=1$.

Provided that $B_{l}^{(N)}, \phi_{j}^{(l)}, \psi_{j}^{(l)}, \phi_{m+i}^{(l)}, \psi_{m+i}^{(l)}$ satisfy (11b) for $l \leq$ $N-1$,

$$
\begin{aligned}
\phi_{\tau_{k}}^{(l)} & =\left[\left(B_{k}^{(l)}+\sum_{j=1}^{m+l} \phi_{j}^{(l)} \partial_{q}^{-1} \psi_{j}^{(l)}\right) \phi^{(l)}\right], \\
b_{j}\left(\tau_{k}\right)_{\tau_{k}}=(-1)^{(l+i)} \beta_{i}\left(\tau_{k}\right) \eta_{i}\left(\tau_{k}\right), & \\
& \quad l=1, \ldots, N-1 .
\end{aligned}
$$

Noticing that $\phi^{(N)}=\left[D_{N} \phi^{(N-1)}\right]$, then when $l=N$, we have

$$
\begin{aligned}
\phi_{\tau_{k}}^{(N)}- & {\left[\left(B_{k}^{(N)}+\sum_{j=1}^{m+N} \phi_{j}^{(N)} \partial_{q}^{-1} \psi_{j}^{(N)}\right) \phi^{(N)}\right] } \\
= & {\left[D_{N} \phi^{(N-1)}\right]_{\tau_{k}}-\left[\left(B_{k}^{(N)} D_{N}\right.\right.} \\
& \left.\left.+\sum_{j=1}^{m+N} \phi_{j}^{(N)} \partial_{q}^{-1} \psi_{j}^{(N)} D_{N}\right) \phi^{(N-1)}\right]=\left[\left(D_{N, \tau_{k}}\right.\right. \\
+ & D_{N} B_{k}^{(N-1)}-B_{k}^{(N)} D_{N} \\
+ & \sum_{j=1}^{m+N-1} D_{N} \phi_{j}^{(N-1)} \partial_{q}^{-1} \psi_{j}^{(N-1)} \\
& \left.\left.-\sum_{j=1}^{m+N} \phi_{j}^{(N)} \partial_{q}^{-1} \psi_{j}^{(N)} D_{N}\right) \phi^{(N-1)}\right]
\end{aligned}
$$

simplifying $\quad \sum_{j=1}^{m+N-1} D_{N} \phi_{j}^{(N-1)} \partial_{q}^{-1} \psi_{j}^{(N-1)}$ $\sum_{j=1}^{m+N} \phi_{j}^{(N)} \partial_{q}^{-1} \psi_{j}^{(N)} D_{N}$ leads to

$$
\begin{aligned}
& \sum_{j=1}^{m+N-1} D_{N} \phi_{j}^{(N-1)} \partial_{q}^{-1} \psi_{j}^{(N-1)}-\sum_{j=1}^{m+N} \phi_{j}^{(N)} \partial_{q}^{-1} \psi_{j}^{(N)} D_{N} \\
& =\sum_{j=1}^{m+N-1}\left(D_{N} \phi_{j}^{(N-1)} \partial_{q}^{-1} \psi_{j}^{(N-1)}-\phi_{j}^{(N)} \partial_{q}^{-1} \psi_{j}^{(N)} D_{N}\right) \\
& \quad-\phi_{m+N}^{(N)} \partial_{q}^{-1} \psi_{m+N}^{(N)} D_{N} .
\end{aligned}
$$

From (40f), we obtain

$$
\begin{aligned}
\psi_{m+N}^{(N)} & =(-1)^{m+N} \eta_{N}\left(\tau_{k}\right) \frac{\theta\left(W_{q}\left[h_{1}, h_{2}, \ldots, h_{i-1}\right]\right)}{\theta\left(W_{q}\left[h_{1}, h_{2}, \ldots, h_{N}\right]\right)} \\
& =(-1)^{m+N} \frac{\eta_{N}\left(\tau_{k}\right)}{\theta\left(\left[T_{N-1} h_{N}\right]\right)} \\
& =(-1)^{m+N} \frac{\eta_{N}\left(\tau_{k}\right)}{\theta\left(h_{N}^{(N-1)}\right)} .
\end{aligned}
$$

Substituting (44b) into (44a) yields

$$
\begin{aligned}
& \sum_{j=1}^{m+N-1} D_{N} \phi_{j}^{(N-1)} \partial_{q}^{-1} \psi_{j}^{(N-1)}-\sum_{j=1}^{m+N} \phi_{j}^{(N)} \partial_{q}^{-1} \psi_{j}^{(N)} D_{N} \\
&=\sum_{j=1}^{m+N-1}\left(D_{N} \phi_{j}^{(N-1)} \partial_{q}^{-1} \psi_{j}^{(N-1)}-\phi_{j}^{(N)} \partial_{q}^{-1} \psi_{j}^{(N)} D_{N}\right) \\
& \quad-(-1)^{m+N} \frac{\eta_{N}\left(\tau_{k}\right) \phi_{m+N}^{(N)}}{h_{N}^{(N-1)}} \\
&+(-1)^{m+N} \eta_{N}\left(\tau_{k}\right) \phi_{m+N}^{(N)} \partial_{q}^{-1}\left[\partial_{q} \frac{1}{h_{N}^{(N-1)}}\right] \\
&+\phi_{m+N}^{(N)} \partial_{q}^{-1} \psi_{m+N}^{(N)} \alpha_{N}^{N-1} \\
&=\sum_{j=1}^{m+N-1}\left(D_{N} \phi_{j}^{(N-1)} \partial_{q}^{-1} \psi_{j}^{(N-1)}-\phi_{j}^{(N)} \partial_{q}^{-1} \psi_{j}^{(N)} D_{N}\right) \\
&-(-1)^{m+N} \eta_{N}\left(\tau_{k}\right) \phi_{m+N}^{(N)} \partial_{q}^{-1} \frac{\left[\partial_{q} h_{N}^{(N-1)}\right]}{\theta\left(h_{N}^{(N-1)}\right) h_{N}^{(N-1)}} \\
&+\phi_{m+N}^{(N)} \partial_{q}^{-1} \psi_{m+N}^{(N)} \alpha_{N}^{N-1} \\
&= \sum_{j=1}^{m+N-1}\left(D_{N} \phi_{j}^{(N-1)} \partial_{q}^{-1} \psi_{j}^{(N-1)}-\phi_{j}^{(N)} \partial_{q}^{-1} \psi_{j}^{(N)} D_{N}\right) \\
&+(-1)^{m+N} \frac{\beta_{N}\left(\tau_{k}\right) \eta_{N}\left(\tau_{k}\right)\left[T_{N} g_{N}\right]}{h_{N}^{(N-1)}} .
\end{aligned}
$$


From (37a) for one DBT $D_{N}$, we have

$$
\begin{aligned}
& \sum_{j=1}^{m+N-1}\left(D_{N} \phi_{j}^{(N-1)} \partial_{q}^{-1} \psi_{j}^{(N-1)}-\sum_{j=1}^{m+N} \phi_{j}^{(N)} \partial_{q}^{-1} \psi_{j}^{(N)} D_{N}\right) \\
& =\frac{1}{h_{N}^{(N-1)}}\left[D _ { N } \left[\sum_{j=1}^{m+N-1}\left(\phi_{j}^{(N-1)} \partial_{q}^{-1} \psi_{j}^{(N-1)}\right)\right.\right. \\
& \left.\left.\cdot h_{N}^{(N-1)}\right]\right] .
\end{aligned}
$$

Note that $h_{N}^{(N-1)}$ satisfies

$$
\begin{aligned}
& h_{N, \tau_{k}}^{(N-1)} \\
& =\left[\left(B_{k}^{(N-1)}+\sum_{j=1}^{m+N-1} \phi_{j}^{(N-1)} \partial_{q}^{-1} \psi_{j}^{(N-1)}\right) h_{N}^{(N-1)}\right] \\
& \quad+b_{N}\left(\tau_{k}\right)_{\tau_{k}} g_{N}^{(N-1)} \Longrightarrow \\
& {\left[\left(\sum_{j=1}^{m+N-1} \phi_{j}^{(N-1)} \partial_{q}^{-1} \psi_{j}^{(N-1)}\right) h_{N}^{(N-1)}\right]} \\
& \quad=h_{N, \tau_{k}}^{(N-1)}-\left[B_{k}^{(N-1)} h_{N}^{(N-1)}\right]-b_{N}\left(\tau_{k}\right)_{\tau_{k}} g_{N}^{(N-1)},
\end{aligned}
$$

and that

$$
\left[T_{N} h_{N}\right]=\left[D_{N}\left[T_{N-1} h_{N}\right]\right]=\left[D_{N} h_{N}^{(N-1)}\right]=0 .
$$

Differentiating both sides of (47b) with respect to $\tau_{k}$ yields

$$
\begin{aligned}
{\left[D_{N, \tau_{k}} h_{N}^{(N-1)}\right]+\left[D_{N} h_{N, \tau_{k}}^{(N-1)}\right] } & =0 \Longrightarrow \\
{\left[D_{N} h_{N, \tau_{k}}^{(N-1)}\right] } & =-\left[D_{N, \tau_{k}} h_{N}^{(N-1)}\right] ;
\end{aligned}
$$

we obtain

$$
\begin{aligned}
& \frac{1}{h_{N}^{(N-1)}}\left[D_{N}\left[\sum_{j=1}^{m+N-1}\left(\phi_{j}^{(N-1)} \partial_{q}^{-1} \psi_{j}^{(N-1)}\right) h_{N}^{(N-1)}\right]\right] \\
& =-\frac{\left[B_{k}^{(N-1)}\left[D_{N} h_{N}^{(N-1)}\right]\right]}{h_{N}^{(N-1)}}=0 .
\end{aligned}
$$

Combining (43), (45), (46), and (49), we get

$$
\begin{gathered}
\phi_{\tau_{k}}^{(N)}-\left[\left(B_{k}^{(N)}+\sum_{j=1}^{m+N} \phi_{j}^{(N)} \partial_{q}^{-1} \psi_{j}^{(N)}\right) \phi^{(N)}\right] \\
=(-1)^{m+N} \frac{\beta_{N}\left(\tau_{k}\right) \eta_{N}\left(\tau_{k}\right)\left[T_{N} g_{N}\right]}{h_{N}^{(N-1)}} \\
-\frac{b_{N}\left(\tau_{k}\right)_{\tau_{k}}\left[T_{N} g_{N}\right]}{h_{N}^{(N-1)}}=0 .
\end{gathered}
$$

This completes the proof.

\section{Soliton Solution of $q$-KdVHSCS}

It is known that $\mathrm{KdV}$ equation is the first nontrivial equation of the KdV hierarchy. However, the first nontrivial equation of $q$-KdVHSCS is not the $q$-KdVESCS but (12a), (12b), (12c), (12d), and (12e). In this section, we aim to construct the soliton solution to (12a), (12b), (12c), (12d), and (12e). In order to get the soliton solution of (12a), (12b), (12c), (12d), and (12e), the following proposition is firstly presented.

Proposition 5. Let $f_{1}, g_{1}$ be two independent wave functions of (12e), $h_{1} \equiv f_{1}+b_{1}\left(\tau_{1}\right) g_{1}$, under the nonauto DBT, and the transformed coefficients are given by

$$
\bar{v}_{1}-v_{1}=x(q-1)\left(\bar{v}_{0}-v_{0}\right)
$$

where

$$
\begin{aligned}
\bar{v}_{0} & =v_{0}+\left[\partial _ { q } \left(\theta\left(\frac{f_{1, \tau_{1}}+b_{1}\left(\tau_{1}\right) g_{1, \tau_{1}}}{h_{1}}\right)\right.\right. \\
& \left.\left.+\frac{f_{1, \tau_{1}}+b_{1}\left(\tau_{1}\right) g_{1, \tau_{1}}}{h_{1}}\right)\right] .
\end{aligned}
$$

Proof. It was shown in [7] that formula (51) holds for (12a), (12b), (12c), (12d), and (12e) and that

$$
\bar{v}_{0}-v_{0}=\left[\partial_{q}\left(v_{1}+\alpha_{1}+\theta\left(\alpha_{1}\right)\right)\right] .
$$

Noting that $h_{1}=f_{1}+b_{1}\left(\tau_{1}\right) g_{1},\left(B_{2}\right)_{\geq 0}^{1 / 2}=B_{1}=\partial_{q}+u_{0}$, then we have

$$
\begin{aligned}
h_{1, \tau_{1}} & =\left[\left(\left(B_{2}\right)_{\geq 0}^{1 / 2}+\sum_{j=1}^{m} \phi_{j} \partial_{q}^{-1} \psi_{j}\right) h_{1}\right]+b_{1}\left(\tau_{1}\right)_{\tau_{1}} g_{1} \\
& =\left[\partial_{q} h_{1}\right]+u_{0} h_{1}+\sum_{j=1}^{m} \phi_{j} \Omega\left(\psi_{j}, h_{1}\right)+b_{1}\left(\tau_{1}\right)_{\tau_{1}} g_{1} .
\end{aligned}
$$

From (54), we get

$$
\begin{aligned}
u_{0}= & \frac{h_{1, \tau_{1}}-\left[\partial_{q} h_{1}\right]}{h_{1}}-\frac{1}{h_{1}} \sum_{j=1}^{m} \phi_{j} \Omega\left(\psi_{j}, h_{1}\right) \\
& -b_{1}\left(\tau_{1}\right)_{\tau_{1}} \frac{g_{1}}{h_{1}} .
\end{aligned}
$$

Noticing that (12c) implies

$$
v_{1}=\theta\left(u_{0}\right)+u_{0}
$$


we have

$$
\begin{aligned}
& \bar{v}_{0}-v_{0}=\left[\partial _ { q } \left(\theta\left(\frac{h_{1, \tau_{1}}-\left[\partial_{q} h_{1}\right]}{h_{1}}\right)+\frac{h_{1, \tau_{1}}-\left[\partial_{q} h_{1}\right]}{h_{1}}\right.\right. \\
& -\theta\left(\frac{1}{h_{1}} \sum_{j=1}^{m} \phi_{j} \Omega\left(\psi_{j}, h_{1}\right)+b_{1}\left(\tau_{1}\right)_{\tau_{1}} \frac{g_{1}}{h_{1}}\right) \\
& -\left(\frac{1}{h_{1}} \sum_{j=1}^{m} \phi_{j} \Omega\left(\psi_{j}, h_{1}\right)+b_{1}\left(\tau_{1}\right)_{\tau_{1}} \frac{g_{1}}{h_{1}}\right)+\frac{\left[\partial_{q} h_{1}\right]}{h_{1}} \\
& \left.\left.+\theta\left(\frac{\left[\partial_{q} h_{1}\right]}{h_{1}}\right)\right)\right]=\left[\partial_{q}\left(\theta\left(\frac{h_{1, \tau_{1}}}{h_{1}}\right)\right)+\frac{h_{1, \tau_{1}}}{h_{1}}\right. \\
& -\theta\left(\frac{1}{h_{1}} \sum_{j=1}^{m} \phi_{j} \Omega\left(\psi_{j}, h_{1}\right)+b_{1}\left(\tau_{1}\right)_{\tau_{1}} \frac{g_{1}}{h_{1}}\right) \\
& \left.-\left(\frac{1}{h_{1}} \sum_{j=1}^{m} \phi_{j} \Omega\left(\psi_{j}, h_{1}\right)+b_{1}\left(\tau_{1}\right)_{\tau_{1}} \frac{g_{1}}{h_{1}}\right)\right] \\
& =\left[\partial _ { q } \left(\theta\left(\frac{f_{1, \tau_{1}}+b_{1}\left(\tau_{1}\right) g_{1, \tau_{1}}}{h_{1}}\right)\right.\right. \\
& \left.\left.+\frac{f_{1, \tau_{1}}+b_{1}\left(\tau_{1}\right) g_{1, \tau_{1}}}{h_{1}}\right)\right] \\
& -\left[\partial _ { q } \left(\theta\left(\frac{1}{h_{1}} \sum_{j=1}^{m} \phi_{j} \Omega\left(\psi_{j}, h_{1}\right)\right)\right.\right. \\
& \left.\left.+\left(\frac{1}{h_{1}} \sum_{j=1}^{m} \phi_{j} \Omega\left(\psi_{j}, h_{1}\right)\right)\right)\right] \\
& =\left[\partial _ { q } \left(\theta\left(\frac{f_{1, \tau_{1}}+b_{1}\left(\tau_{1}\right) g_{1, \tau_{1}}}{h_{1}}\right)\right.\right. \\
& \left.\left.+\frac{f_{1, \tau_{1}}+b_{1}\left(\tau_{1}\right) g_{1, \tau_{1}}}{h_{1}}\right)\right]+(q \theta+1) \\
& \cdot\left[\partial_{q}\left(\frac{1}{h_{1}} \sum_{j=1}^{m} \phi_{j} \Omega\left(\psi_{j}, h_{1}\right)\right)\right] .
\end{aligned}
$$

Next we consider

$$
\begin{aligned}
& {\left[\partial_{q}\left(\frac{1}{h_{1}} \sum_{j=1}^{m} \phi_{j} \Omega\left(\psi_{j}, h_{1}\right)\right)\right]} \\
& =\sum_{j=1}^{m}\left(\theta\left(\frac{\phi_{j}}{h_{1}}\right)\left[\partial_{q} \Omega\left(\psi_{j}, h_{1}\right)\right]\right. \\
& \left.+\left[\partial_{q} \frac{\phi_{j}}{h_{1}}\right] \Omega\left(\psi_{j}, h_{1}\right)\right)=\sum_{j=1}^{m}\left(\frac{\theta\left(\phi_{j}\right) \psi_{j} h_{1}}{\theta\left(h_{1}\right)}\right.
\end{aligned}
$$

$$
\begin{aligned}
& \left.+\frac{\left[\partial_{q} \phi_{j}\right] h_{1}-\phi_{j}\left[\partial_{q} h_{1}\right]}{\theta\left(h_{1}\right) h_{1}} \Omega\left(\psi_{j}, h_{1}\right)\right)=\frac{1}{\theta\left(h_{1}\right)} \\
& \cdot \sum_{j=1}^{m}\left(\theta\left(\phi_{j}\right) \psi_{j} h_{1}+\left(\left[\partial_{q} \phi_{j}\right]-\alpha_{1} \phi_{j}\right) \Omega\left(\psi_{j}, h_{1}\right)\right) \\
& =\frac{h_{1}}{\theta\left(h_{1}\right)}\left(\sum_{j=1}^{m} \theta\left(\phi_{j}\right) \psi_{j}+\bar{\phi}_{j} \frac{\Omega\left(\psi_{j}, h_{1}\right)}{h_{1}}\right)
\end{aligned}
$$

Noting (37b), we can immediately derive

$$
\left[\partial_{q}\left(\frac{1}{h_{1}} \sum_{j=1}^{m} \phi_{j} \Omega\left(\psi_{j}, h_{1}\right)\right)\right]=0 .
$$

Hence we obtain from (57)

$$
\begin{aligned}
\bar{v}_{0} & =v_{0}+\left[\partial _ { q } \left(\theta\left(\frac{f_{1, \tau_{1}}+b_{1}\left(\tau_{1}\right) g_{1, \tau_{1}}}{h_{1}}\right)\right.\right. \\
& \left.\left.+\frac{f_{1, \tau_{1}}+b_{1}\left(\tau_{1}\right) g_{1, \tau_{1}}}{h_{1}}\right)\right] .
\end{aligned}
$$

This completes the proof.

Next we will start from the trivial solution to (12a), (12b), (12c), (12d), and (12e) without sources, that is, $v_{0}=v_{1}=0$, and use Theorem 3 and Proposition 5 to construct one soliton solution to (12a), (12b), (12c), (12d), and (12e) with $m=1$. When $v_{0}=v_{1}=0$, then $B_{2}=\partial_{q}^{2}$; hence, the wave functions $f_{1}, g_{1}$ of Lax operator $B_{2}=\partial_{q}^{2}$ satisfy

$$
\begin{aligned}
{\left[\partial_{q}^{2} \varphi\right] } & =\lambda_{1}^{2} \varphi, \\
\phi_{\tau_{1}} & =\left[\partial_{q} \varphi\right] .
\end{aligned}
$$

We take the solution $f_{1}, g_{1}$ of system (61) as follows:

$$
\begin{aligned}
& f_{1}=E_{q}\left(p_{1} x\right) \exp \left(p_{1} \tau_{1}\right), \\
& g_{1}=E_{q}\left(-p_{1} x\right) \exp \left(-p_{1} \tau_{1}\right),
\end{aligned}
$$

where $E_{q}(x)$ denotes the $q$-exponential function satisfying

$$
\left[\partial_{q} E_{q}\left(p_{1} x\right)\right]=p_{1} E_{q}\left(p_{1} x\right),
$$

with an equivalent form

$$
E_{q}(x)=\sum_{k=0}^{\infty} \frac{1}{[k]_{q} !} x^{k} .
$$


Noting $h_{1} \equiv f_{1}+b_{1}\left(\tau_{1}\right) g_{1}$, where $f_{1}, g_{1}$ are defined by (62), we get from (51) and (52)

$$
\begin{aligned}
& \bar{v}_{0} \\
& =\frac{p_{1}\left(h_{1} \theta^{2}\left(f_{1}-b_{1}\left(\tau_{1}\right) g_{1}\right)-\left(f_{1}-b_{1}\left(\tau_{1}\right) g_{1}\right) \theta^{2}\left(h_{1}\right)\right)}{x(q-1) h_{1} \theta^{2}\left(h_{1}\right)}, \\
& \bar{v}_{1} \\
& =\frac{p_{1}\left(h_{1} \theta^{2}\left(f_{1}-b_{1}\left(\tau_{1}\right) g_{1}\right)-\left(f_{1}-b_{1}\left(\tau_{1}\right) g_{1}\right) \theta^{2}\left(h_{1}\right)\right)}{h_{1} \theta^{2}\left(h_{1}\right)} .
\end{aligned}
$$

In addition, by Theorem 3, we obtain

$$
\begin{aligned}
& \bar{\phi}_{1}=-\beta_{1}\left(\tau_{1}\right) \frac{h_{1}\left[\partial_{q} g_{1}\right]-g_{1}\left[\partial_{q} h_{1}\right]}{h_{1}^{2}}, \\
& \bar{\psi}_{1}=-\frac{\eta_{1}\left(\tau_{1}\right)}{\theta_{1}\left(h_{1}\right)}
\end{aligned}
$$

where $b_{1}\left(\tau_{1}\right), \beta_{1}\left(\tau_{1}\right)$, and $\eta_{1}\left(\tau_{1}\right)$ satisfy

$$
b_{1}\left(\tau_{1}\right)_{\tau_{1}}=-\beta_{q}\left(\tau_{1}\right) \eta_{1}\left(\tau_{1}\right) .
$$

Then (65a), (65b), (65c), (65d), and (65e) present one soliton solution of (12a), (12b), (12c), (12d), and (12e) with $m=$ 1. In particular, when $b_{1}\left(\tau_{1}\right)=c$, where $c$ is an arbitrary constant, (65a), (65b), (65c), (65d), and (65e) can be reduced to one soliton solution to the first nontrivial equation of the $q$-KdV hierarchy [7]. Certainly, we also use Theorem 4 and Proposition 5 to construct the multisoliton solution to (12a), (12b), (12c), (12d), and (12e). But owing to the complexity of the computation, we omit it here.

\section{Summary}

As $n$-reduction of the extended $q$-deformed KP hierarchy, $q$ NKdVHSCS is explored in this paper. Two kinds of DBTs are constructed and the soliton solution to the first nontrivial equation of $q$-KdVHSCS is also obtained. We find that one of the DBTs provides a nonauto Bäcklund transformation for the two $q$-NKdVESCS with different degree, which enables us to obtain the new solution of $q$-NKdVHSCS from the known solution of $q-\mathrm{NKdVH}$. Noting that we only investigate DBT and solution of $q$-NKdVESCS, other integrable structures will be studied in our forthcoming paper such as infinite conservation law, tau function, and Hamiltonian structure.

\section{Competing Interests}

The authors declare that there are no competing interests regarding the publication of this paper.

\section{Acknowledgments}

This work is supported by National Natural Science Foundation of China (Grant nos. 11201178 and 11171175), Fujian National Science Foundation (Grant no. 2012J01013), Fujian
Higher College Special Project of Scientfic Research (Grant no. JK2012025), Fujian provincial visiting scholar program, and the Scientific Research Foundation of Jimei University, China.

\section{References}

[1] A. Klimyk and K. Schmudgen, " $q$-calculus," in Quantum Groups and their Represntaions, pp. 37-52, Springer, Berlin, Germany, 1997.

[2] Z. Y. Wu, D. H. Zhang, and Q. R. Zheng, "Quantum deformation of $\mathrm{KdV}$ hierarchies and their exact solutions: $q$-deformed solitons," Journal of Physics A: Mathematical and General, vol. 27, no. 15, pp. 5307-5312, 1994.

[3] E. Frenkel and N. Reshetikhin, "Quantum affine algebras and deformations of the Virasoro and W-algebras," Communications in Mathematical Physics, vol. 178, no. 1, pp. 237-264, 1996.

[4] E. Frenkel, "Deformations of the KdV hierarchy and related soliton equations," International Mathematics Research Notices, no. 2, pp. 55-76, 1996.

[5] L. Haine and P. Iliev, "The bispectral property of a $q$ deformation of the Schur polynomials and the $q-\mathrm{KdV}$ hierarchy," Journal of Physics A: Mathematical and General, vol. 30, no. 20 , pp. 7217-7227, 1997.

[6] M. Adler, E. Horozov, and P. van Moerbeke, "The solution to the q-KdV equation," Physics Letters A, vol. 242, no. 3, pp. 139-151, 1998.

[7] M.-H. Tu, J.-C. Shaw, and C.-R. Lee, "On Darboux-Bäcklund transformations for the $q$-deformed Korteweg-de Vries hierarchy," Letters in Mathematical Physics, vol. 49, no. 1, pp. 33-45, 1999.

[8] M.-H. Tu and C.-R. Lee, "On the $q$-deformed modified Korteweg-de Vries hierarchy," Physics Letters. A, vol. 266, no. 2-3, pp. 155-159, 2000.

[9] J. Mas and M. Seco, "The algebra of $q$-pseudodifferential symbols and $W_{K P}^{(N)}$-algebra," Journal of Mathematical Physics, vol. 37, pp. 6510-6529, 1996.

[10] P. Iliev, "Tau function solutions to a $q$-deformation of the KP hierarchy," Letters in Mathematical Physics, vol. 44, no. 3, pp. 187-200, 1998.

[11] P. Iliev, " $q-\mathrm{KP}$ hierarchy, bispectrality and Calogero-Moser systems," Journal of Geometry and Physics, vol. 35, no. 2-3, pp. 157-182, 2000.

[12] M.-H. Tu, " $q$-deformed KP hierarchy: its additional symmetries and infinitesimal Bäcklund transformations," Letters in Mathematical Physics, vol. 49, no. 2, pp. 95-103, 1999.

[13] J. S. He, Y. H. Li, and Y. Cheng, " $q$-deformed KP hierarchy and $q$-deformed constrained KP hierarchy," Symmetry, Integrability and Geometry. Methods and Applications, vol. 2, no. 60, p. 32, 2006.

[14] J. S. He, Y. H. Li, and Y. Cheng, " $q$-deformed Gelfand-Dickey hierarchy and the determinant representation of its gauge transformation," Chinese Annals of Mathematics A, vol. 25, no. 3, pp. 373-382, 2004.

[15] K. L. Tian, J. S. He, Y. C. Su, and Y. Cheng, "String equations of the $q$-KP hierarchy," Chinese Annals of Mathematics B, vol. 32, no. 6, pp. 895-904, 2011.

[16] R. L. Lin, X. J. Liu, and Y. B. Zeng, "A new extended $q$-deformed KP hierarchy," Journal of Nonlinear Mathematical Physics, vol. 15, no. 3, pp. 333-347, 2008. 
[17] R. L. Lin, H. Peng, and M. Mañas, "The q-deformed mKP hierarchy with self-consistent sources, Wronskian solutions and solitons," Journal of Physics A: Mathematical and Theoretical, vol. 43, Article ID 434022, 2010. 


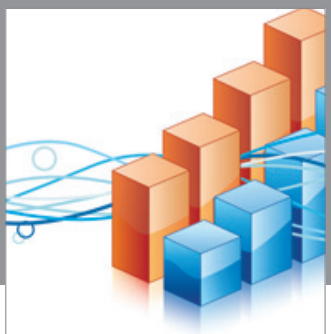

Advances in

Operations Research

vatem alat4

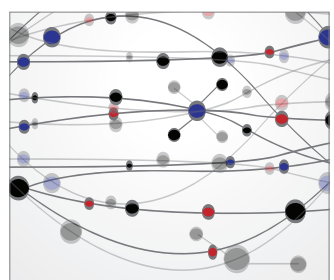

\section{The Scientific} World Journal
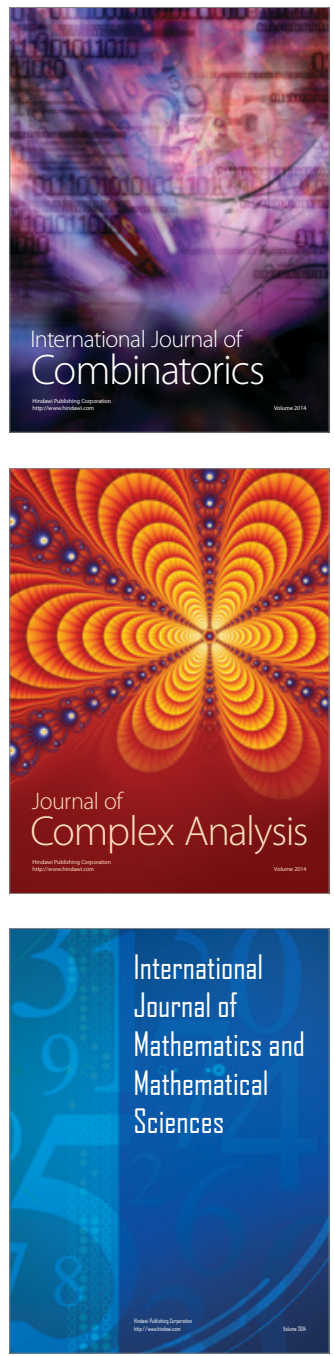
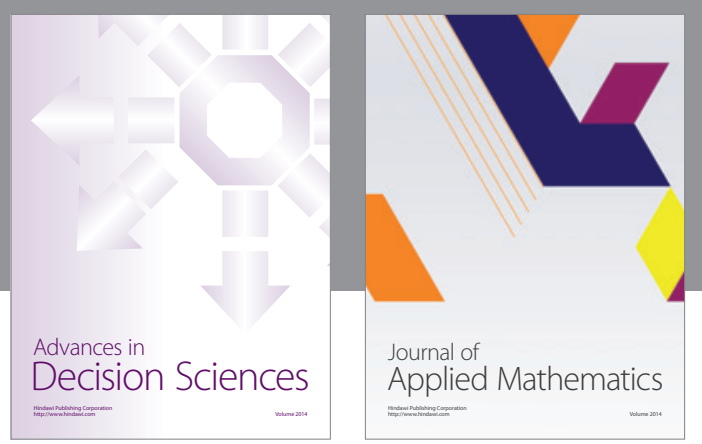

Algebra

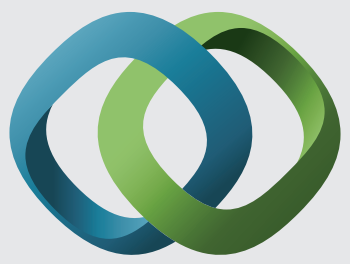

\section{Hindawi}

Submit your manuscripts at

http://www.hindawi.com
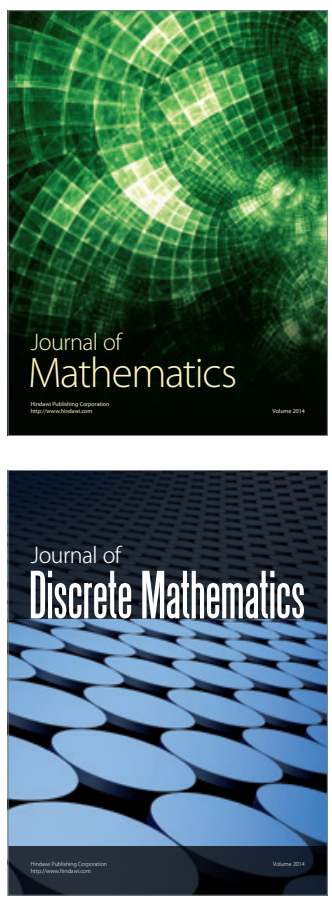

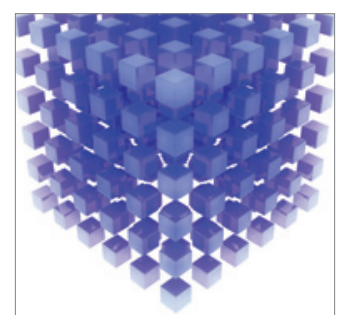

Mathematical Problems in Engineering
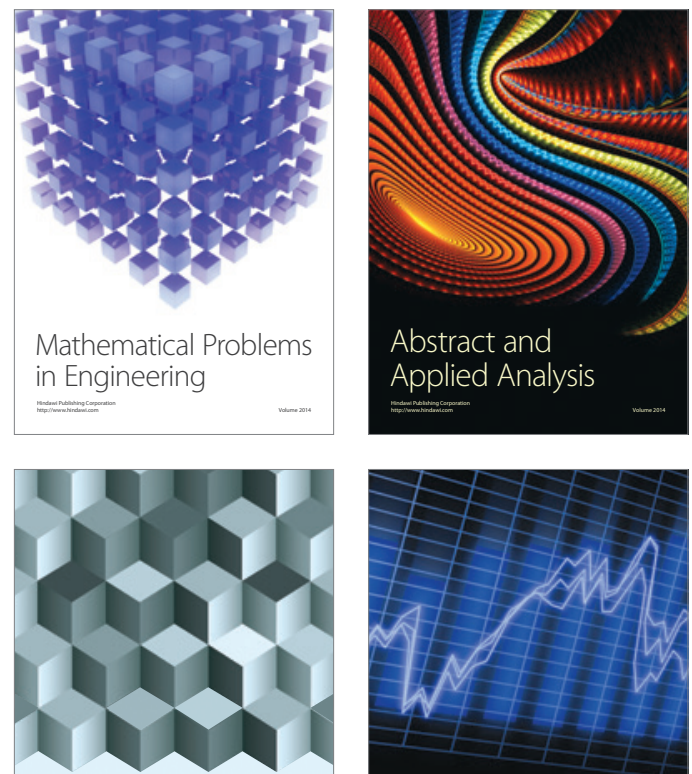

Journal of

Function Spaces

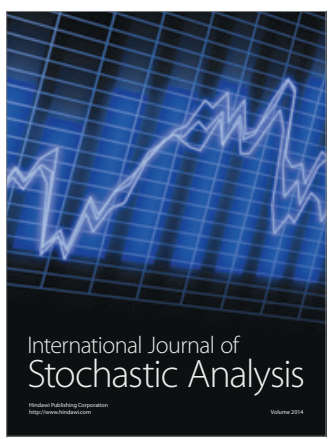

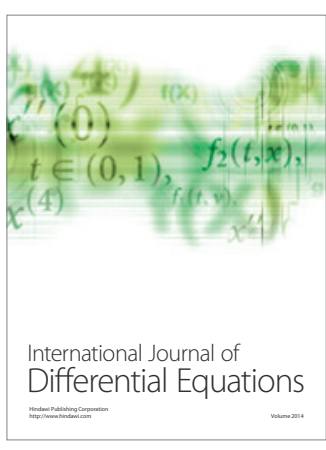
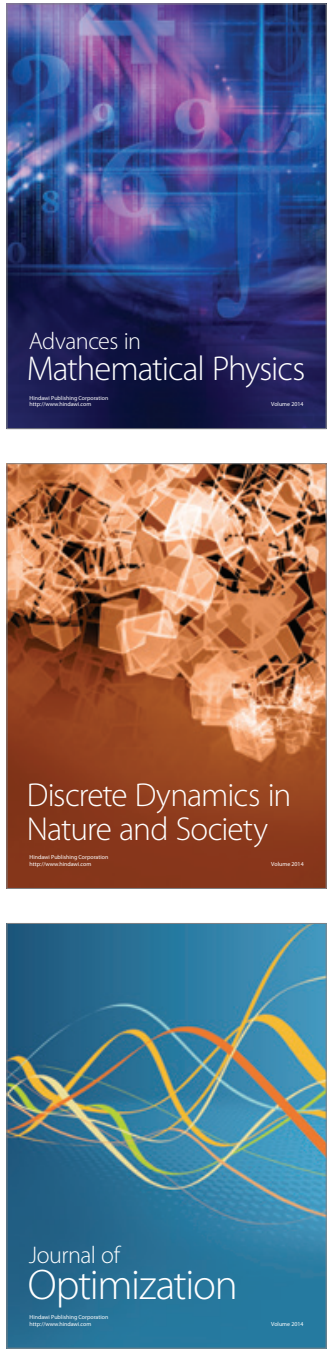